\title{
BENIGN BREAST TUMORS: EPIDEMIOLOGICAL PROFILE AND THERAPEUTIC CONDUCT AT THE ESCOLA PAULISTA DE MEDICINA
}

João Bueno Vitor Peixoto1; Joaquim Teodoro Araújo Neto1, Simone Elias¹, Afonso Celso Pinto Nazário1, Gil Facina

${ }^{1}$ Universidade Federal de São Paulo - São Paulo (SP), Brazil.

Introduction: Breasts represent an important site for the development of diseases. It is known that $80 \%$ of the palpable masses of the breast are of benign origin. Benign breast diseases range from inflammatory to neoplastic processes.

Objectives: to evaluate the clinical and epidemiological characteristics of patients diagnosed with benign breast tumor at the Mastology outpatient clinic of the Universidade Federal de São Paulo (UNIFESP). Methods: 1,532 medical records, available on the electronic platform PEP-HUHSP through the ICD D24 (benign breast cancer) and N63 (unspecified breast nodule), of patients referred to the service between July 2008 and July 2017 were reviewed. After applying exclusion criteria, 403 medical records were submitted to data collection and tabulation in Excel, followed by statistical analysis using the IBM SPSS Statistics 23 software. The study in question was approved by the Research Ethics Committee of UNIFESP (CEP UNIFESP), in the Teaching and Research Coordination of Hospital São Paulo - Hospital Universitário/UNIFESP (CoEP of HSP-HU/UNIFESP) and exempted from the application of the Informed Consent by the same organs. Results: In the 9-year period, the following results were obtained: mean age was 39.3 years. Comorbidities: smoking (16.4\%), SAH (16.8\%) and dyslipidemia (6.3\%). Family history of breast and/or ovarian cancer accounted for 16.6\%. Mean age of menarche and menopause, respectively, were 12.7 and 42.5 years. Causes of referral: "alteration in image examination" (38.3\%), "lump in the breast" (33.3\%), "follow-up due to previous nodules" (16.5\%). Anatomopathological report: fibroadenoma (41\%), breast cysts (16\%), phylloid tumors (3\%), and papilloma (1\%). Mean number of consultations per patient until discharge or abandonment of follow-up: three. Choice behavior: expectant (85.2\%). Conclusion: The epidemiological profile of patients referred to the UNIFESP tertiary mastology service was mainly composed of women of childbearing age and nulliparous women, whose main comorbidities were smoking and SAH, in the great majority with no family history of breast cancer. Regarding the consultation, the main reason for referral is the findings on imaging exams, and, specially, patients would bring their breast USG along, which surpassed mammography by $34.1 \%$. The choice for biopsy was restricted, present in approximately $1 / 3$ of the cases, but pointed out that the most prevalent nodule is fibroadenoma, followed by phylloid and papilloma tumors. Nevertheless, there was a predilection for expectant conduct. On average, there were regular follow-ups for 1.5 years, followed by a significant dropout rate. 